OPEN ACCESS

Edited by:

David J. Seiffge,

University Hospital Bern, Switzerland

Reviewed by:

Hugo Ten Cate,

Maastricht University Medical

Centre, Netherlands

Simona Lattanzi,

Marche Polytechnic University, Italy

${ }^{*}$ Correspondence:

Fadi Nahab

fnahab@emory.edu

Specialty section: This article was submitted to

Stroke,

a section of the journal

Frontiers in Neurology

Received: 14 April 2021

Accepted: 10 May 2021

Published: 07 June 2021

Citation:

Patel K, Mikhael E, Liu M, Rangaraju S, Ellis $D$, Duncan $A$, Belagaje $S$, Belair T, Henriquez $L$ and Nahab F (2021) Anticoagulation Therapy Reduces Recurrent Stroke in

Embolic Stroke of Undetermined

Source Patients With Elevated Coagulation Markers or Severe Left Atrial Enlargement.

Front. Neurol. 12:695378.

doi: 10.3389/fneur.2021.695378

\section{Anticoagulation Therapy Reduces Recurrent Stroke in Embolic Stroke of Undetermined Source Patients With Elevated Coagulation Markers or Severe Left Atrial Enlargement}

\author{
Kishan Patel ${ }^{1}$, Elio Mikhael ${ }^{2}$, Michael Liu ${ }^{3}$, Srikant Rangaraju ${ }^{4}$, Deandra Ellis ${ }^{5}$, \\ Alexander Duncan ${ }^{6}$, Samir Belagaje ${ }^{5}$, Trina Belair ${ }^{5}$, Laura Henriquez ${ }^{5}$ and Fadi Nahab ${ }^{5 *}$ \\ ${ }^{1}$ Department of Neurology, Providence St. Joseph Health, Portland, OR, United States, ${ }^{2}$ Department of Medicine, \\ Saint-Joseph University, Beirut, Lebanon, ${ }^{3}$ Department of Neurology, Mayo Clinic, Rochester, MN, United States, \\ ${ }^{4}$ Department of Neurology, Emory University, Atlanta, GA, United States, ${ }^{5}$ Department of Neurology and Pediatrics, Emory \\ University, Atlanta, GA, United States, ${ }^{6}$ Department of Pathology \& Laboratory Medicine, Emory University, Atlanta, GA, \\ United States
}

Background: The objective of this study was to evaluate if anticoagulation therapy reduces recurrent stroke in embolic stroke of undetermined source (ESUS) patients with left atrial enlargement (LAE) or abnormal markers of coagulation and hemostatic activity (MOCHA) compared to antiplatelet therapy.

Methods: ESUS patients from January 1, 2017, to June 30, 2019, underwent outpatient cardiac monitoring and the MOCHA profile (serum d-dimer, prothrombin fragment 1.2, thrombin-antithrombin complex, and fibrin monomer). Anticoagulation was offered to patients with abnormal MOCHA ( $\geq 2$ elevated markers) or left atrial volume index $\geq 40$ $\mathrm{mL} / \mathrm{m}^{2}$. Patients were evaluated for recurrent stroke or major hemorrhage at routine clinical follow-up. We compared this patient cohort (cohort 2) to a historical cohort (cohort 1) who underwent the same protocol but remained on antiplatelet therapy.

Results: Baseline characteristics in cohort $2(n=196$; mean age $=63 \pm 16$ years, 59\% female, 49\% non-White) were similar to cohort $1(n=42)$ except that cohort 2 had less diabetes ( 43 vs. $24 \%, p=0.01$ ) and more tobacco use ( 26 vs. $43 \%, p=0.04$ ). Overall, 45 patients (23\%) in cohort 2 initiated anticoagulation based on abnormal MOCHA or LAE. During mean follow-up of $13 \pm 10$ months, cohort 2 had significantly lower recurrent stroke rates than cohort 1 (14 vs. $3 \%, p=0.009$ ) with no major hemorrhages.

Conclusions: Anticoagulation therapy in a subgroup of ESUS patients with abnormal MOCHA or severe LAE may be associated with a reduced rate of recurrent stroke compared to antiplatelet therapy. A prospective, randomized study is warranted to validate these results.

Keywords: embolic stroke of undetermined source, biomarkers, left atrial volume index, anticoagulation, cryptogenic stroke 


\section{INTRODUCTION}

Approximately one-third of ischemic strokes are categorized as cryptogenic strokes, and estimates suggest that approximately one-sixth of ischemic strokes meet diagnostic criteria for embolic stroke of undetermined source (ESUS) $(1,2)$.

Anticoagulation therapy for ESUS patients has been postulated to prevent recurrent ischemic strokes; however, empiric treatment with anticoagulation for patients with ESUS has not been deemed beneficial in large randomized controlled trials of unselected cohorts $(1,3)$. Secondary analysis of NAVIGATE ESUS (4) demonstrated a beneficial effect of anticoagulation in subset of patients with moderate to severe left atrial enlargement (LAE) (4). Similarly, subgroup analysis of patients enrolled in the Warfarin-Aspirin Recurrent Stroke Study trial with elevated N-terminal pro-brain natriuretic peptide showed a beneficial effect of anticoagulation in prevention of recurrent stroke (5).

Along with markers of LAE, hematological abnormalities are known to be a contributory factor for ESUS patients. The markers of coagulation and hemostatic activity (MOCHA) profile has been shown to identify patients with occult atrial fibrillation, venous thromboembolism, or undiagnosed underlying malignancy $(6,7)$.

The objective of this study was to evaluate if anticoagulation therapy reduces recurrent stroke in ESUS patients who have LAE on echocardiography or abnormal MOCHA profile.

\section{MATERIALS AND METHODS}

\section{Participants}

This is a retrospective analysis of prospectively collected data. Two cohorts were compared for the analysis: a historical cohort (termed cohort 1) of ESUS patients seen at the Emory Clinic from January 1, 2015, to December 31, 2016 (7), and cohort 2 consisted of ESUS patients seen in the Emory Stroke Clinic from January 1,2017 , to June 30, 2019. All patients underwent brain imaging with a computed tomography or magnetic resonance imaging, which demonstrated a non-lacunar brain infarction during the initial evaluation. Patients with large artery atherosclerosis or occlusion, evidence of cardioembolic source after 12 lead-ECG, and $>24 \mathrm{~h}$ cardiac telemetry on initial inpatient evaluation or echocardiography were excluded from the analysis. Patients were included if they were $\geq 18$ years of age and had completed prolonged outpatient cardiac monitoring with either 30-day mobile cardiac outpatient telemetry and/or implantable loop recorder (Reveal LINQ; Medtronic, Minneapolis, MN). All patients underwent MOCHA testing at least 2 weeks after the ESUS as a standard part of the Emory Clinic cryptogenic stroke evaluation. Patients with an elevation of $\geq 2$ markers were deemed abnormal based on prior studies $(6,7)$. Patients with patent foramen ovale (PFO) were included in the analysis.

\footnotetext{
Abbreviations: ESUS, embolic stroke of undetermined source; LAE, left atrial enlargement; MOCHA, markers of coagulation and hemostatic activation; ECG, electrocardiogram; TTE, transthoracic echocardiography; LAVI, left atrial volume index; AHA/ASA, American Heart Association/American Stroke Association; PFO, patent foramen ovale.
}

At the initial stroke clinic visit, all patients were on single antiplatelet therapy.

\section{Echocardiography}

Standard two-dimensional and Doppler transthoracic echocardiography (TTE) was performed on a GE Vivid 7 and E9 (General Electric, Milwaukee, WI) or Philips IE 33 (Philips, Andover, MA). Left atrial echocardiographic parameters were obtained by TTE including left atrial volume index (LAVI) and left atrial diameter. For patients who did not have these parameters reported, LAE was graded by the reviewing board-certified cardiologist.

\section{Markers of Coagulation and Hemostatic Activity}

The MOCHA profile was obtained on patients and included serum levels of $\mathrm{D}$-dimer (reference value $\leq 574$ ), prothrombin fragment 1.2 (reference value $=65-288 \mathrm{pmol} / \mathrm{L}$ ), thrombinantithrombin complex (reference value $=1.0-5.5 \mu \mathrm{g} / \mathrm{L}$ ), and fibrin monomer (reference value $<7 \mu \mathrm{g} / \mathrm{mL}$ ). Serum Ddimer levels were measured by high-sensitivity latex dimer assay (Instrumentation laboratories, Bedford, MA). Prothrombin fragment 1.2 and thrombin-antithrombin complexes were performed using Enzygnost ELISA kit (Siemens Healthcare, Tarrytown, New York, NY). Fibrin monomer was measured by latex immunoassay (Stago, Parsippany, NJ).

\section{Intervention}

In our two cohorts, all patients presented to the Emory Stroke Clinic on a single antiplatelet therapy with aspirin (81$325 \mathrm{mg}$ daily) at first outpatient visit. Patients in cohort 2 with abnormal MOCHA panel ( $\geq 2$ markers elevated) and/or evidence of LAE (LAVI $\geq 40 \mathrm{~cm} / \mathrm{m}^{2}$ ) on echocardiography were offered anticoagulation therapy after a detailed discussion of available evidence and after weighing the risks of hemorrhagic complications against potential benefit of reducing recurrent stroke. Patients who elected to start anticoagulation were treated with apixaban, dabigatran, rivaroxaban, or warfarin according to provider and patient preference. Patients in cohort 2 with normal MOCHA panel and no LAE on echocardiography remained on single antiplatelet therapy. All patients in cohort 1 (historical cohort) remained on antiplatelet medication, regardless of echocardiography and MOCHA panel findings. The study design is presented in Figure 1.

\section{Endpoints}

Patients were evaluated for the endpoints of recurrent stroke or major hemorrhage at routine clinical follow-up as previously reported (6).

\section{Standard Protocol Approvals, Registration, and Patient Consent}

The presented study was approved by the institutional review board of Emory University. 


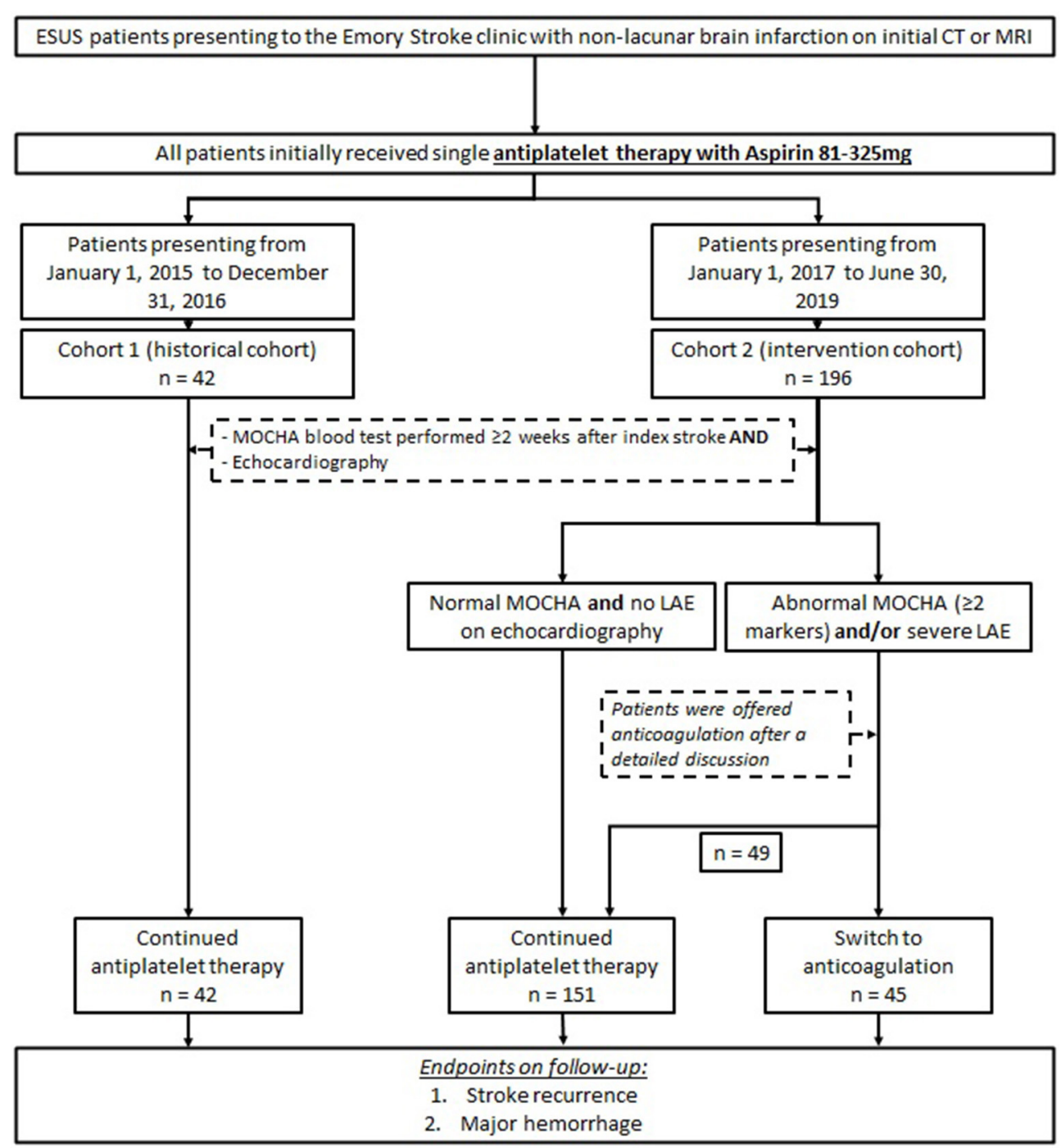

FIGURE 1 | Study design.

\section{Statistical Analysis}

The patients included in this study (cohort 2) were compared to a historical cohort (cohort 1) with abnormal MOCHA who were treated only with antiplatelet therapy during the follow-up period. The cohort 1 data were obtained from a previously published study (7). Independent $t$-tests were used for continuous variables. Categorical variables were compared with Pearson $\chi^{2}$ or Fisher exact test. Non-parametric comparison was performed using the Mann-Whitney $U$-test. Statistical analysis was performed using a combination of programs, including SPSS v25, Vassar Stats, and GraphPad. $p$-Values were calculated, and statistical significance was determined to be $p<0.05$.

\section{RESULTS}

During the study period for cohort 2, 196 patients met the criteria for study inclusion. The mean age was $63 \pm 16$ years,
$59 \%$ were female, and $49 \%$ were non-White. Compared to the historical cohort, the baseline demographic characteristics were similar (Table 1). In cohort 2, there was a lower percentage of patients with diabetes ( 43 vs. $24 \%, p=0.01$ ) but had a higher percentage of patients with any tobacco use (43 vs. $26 \%, p=$ 0.045). Compared to cohort 1 , there was no significant difference between patients with hypertension ( 71 vs. $75 \%, p=0.68$ ) or a history of prior ischemic stroke ( 9.5 vs. $13 \%, p=0.62$ ). In cohort 2, 79 patients (40\%) underwent loop recorder placement compared to 28 patients $(67 \%)$ in cohort 1 . All other patients underwent mobile cardiac outpatient telemetry (Table 1).

Median days to MOCHA testing was 46 [interquartile range $(\mathrm{IQR})=23-88$ ] days compared to 33 (IQR $=15-57)$ days in cohort 1 . There was no significant difference in the frequency of abnormal MOCHA between cohorts with 82 patients (42\%) having abnormal MOCHA ( $\geq 2$ elevated markers) in cohort 2 compared to 23 patients (55\%) in cohort 1 . On echocardiography, 
TABLE 1 | Baseline characteristics.

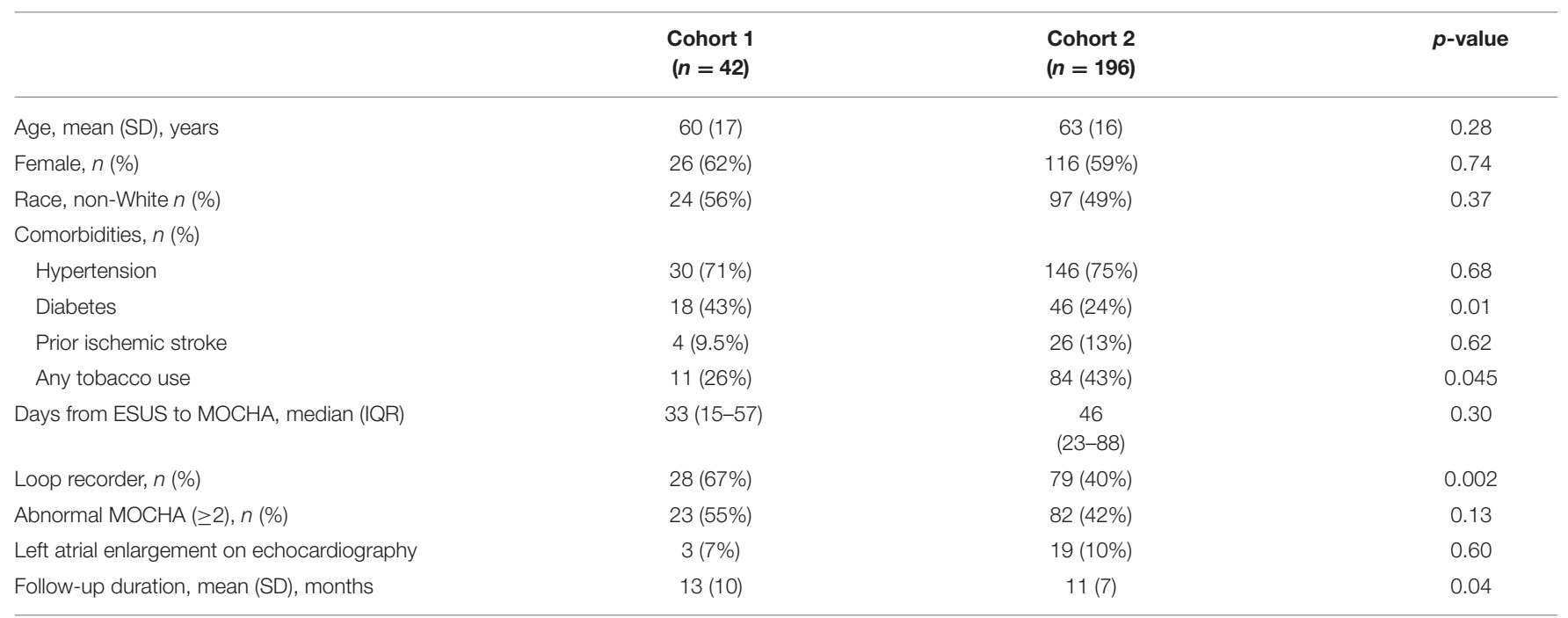

TABLE 2 | Results.

\begin{tabular}{lccc}
\hline & $\begin{array}{c}\text { Cohort 1 } \\
(\boldsymbol{n}=\mathbf{4 2})\end{array}$ & $\begin{array}{c}\text { Cohort 2 } \\
(\boldsymbol{n}=196)\end{array}$ & $\boldsymbol{p}$-value \\
\hline Endpoint, $n(\%)$ & & & 0.009 \\
Recurrent stroke & $6(14 \%)$ & $6(3 \%)$ & 1.0 \\
Major hemorrhage & $0(0 \%)$ & $0(0 \%)$ & \\
\hline
\end{tabular}

19 patients (10\%) met the criteria for severe LAE (LAVI $\geq 40$ $\mathrm{cm} / \mathrm{m}^{2}$ ) in cohort 2 , whereas 3 patients $(7 \%)$ met the same criteria in cohort 1 . The difference was not significant (Table 1).

In cohort 2,45 patients (23\%) were placed on anticoagulation, including 33 patients with abnormal MOCHA profile alone, 8 patients with severe LAE alone, and 4 patients with both abnormal MOCHA profile and severe LAE. In total, 37 patients (82\%) received apixaban, 4 received warfarin, 2 received rivaroxaban, 1 patient received dabigatran, and 1 patient received low-molecular-weight heparin.

During mean follow-up of $13 \pm 10$ months, cohort 2 had significantly lower recurrent stroke rates than cohort 1 (14 vs. $3 \%, p=0.009)$ with no major hemorrhages in either cohort (Table 2). One patient (2\%) on anticoagulation therapy had recurrent stroke, whereas $6(14 \%)$ in the historical cohort had a recurrence $(p=0.04)$.

\section{DISCUSSION}

Our study aimed to explore the potential effects of anticoagulation in a subset of ESUS patients who have evidence of abnormal coagulation and hemostatic activity or LAE on echocardiography. Overall, we found a lower rate of recurrent stroke in a cohort of patients placed on anticoagulation therapy for abnormal MOCHA or severe LAE vs. antiplatelet therapy.
ESUS patients can be subclassified into many categories. Lattanzi et al. identified three subgroups of ESUS patients who might benefit from anticoagulation therapy (8). The first subgroup includes young males with PFO, no vascular risk factors, and posterior circulation strokes. However, a recent subgroup analysis of the RE-SPECT ESUS trial showed that anticoagulation with dabigatran compared to antiplatelet therapy did not result in reduced risk of recurrent stroke in ESUS patients with PFO (9). A second subgroup included patients with history of hypertension, diabetes mellitus, and left atrial cardiopathy with severe strokes involving multiple vascular territories. The third subgroup consisted of smokers with dyslipidemia, carotid atherosclerosis, and anterior circulation strokes (8). In addition, in a group of patients with no evidence of potential embolic source, anticoagulation seems to be superior to antiplatelet therapy (10). Nonetheless, the study is an observational one, and the findings require confirmation in a large-scale trial.

LAE on echocardiography and abnormal elevation in the MOCHA profile does identify a subgroup of ESUS patients who are at increased risk of subsequent diagnosis of atrial fibrillation and occult malignancy, respectively (7). As demonstrated in a prior study, a MOCHA profile and markers of left atrial cardiopathy may also be complementary in identifying causes of stroke in ESUS patients (6). We chose to characterize the left atrial cardiopathy by means of stratifying by LAVI on TTE. LAVI has been shown to be an independent predictor of atrial fibrillation and recurrent stroke (11). This is different than the ongoing ARCADIA trial, which is utilizing left atrial diameter on TTE but is limited by its two-dimensional measurement (12).

The present cohort was compared against a historical cohort of patients who underwent a similar protocol for diagnostic evaluation except that all patients were placed on antiplatelet therapy in cohort 1. Anticoagulation therapy offered to the subset of patients in cohort 2 who had abnormal MOCHA 
or severe LAE likely explains the significant reduction in recurrent stroke seen given that no patients with normal MOCHA or LAVI $<40$ had a recurrent stroke in cohort 2 . Our stratification of treatment based on MOCHA and LAE is different from that of the NAVIGATE ESUS (3) and RESPECT ESUS (1) trials, which randomized ESUS patients to anticoagulation vs. antiplatelet therapy without assessment of coagulation markers or left atrial size. In these ESUS cohorts, both rivaroxaban and dabigatran were associated with an increased bleeding risk compared with antiplatelet therapy without significant reduction in stroke or systemic embolism. However, secondary analyses of NAVIGATE ESUS showed that ESUS patients with a predefined left atrial diameter of more than $4.6 \mathrm{~cm}$ had lower ischemic stroke rates when treated with rivaroxaban vs. aspirin (1.7 vs. $6.5 \%$; hazard ratio $=0.26$; $95 \%$ confidence interval $=0.07-0.94)(2)$. The reduction in ischemic stroke with anticoagulation therapy has to be balanced by the risk of hemorrhagic complication, as evidenced by another secondary analysis of the NAVIGATE ESUS trial, which found that the presence of microbleeds on baseline imaging was associated with increased stroke recurrence and increased brain hemorrhage, independent of antiplatelet or anticoagulation therapy (13).

This study has several limitations. First, it is a retrospective analysis of prospectively enrolled patients with ESUS. Second, anticoagulation treatment for ESUS patients based on coagulation abnormalities and left atrial cardiopathy remains off-label therapy. As a result of that, only $23 \%$ of cohort 2 patients elected to be placed on anticoagulation therapy. The exact treatment effect of anticoagulation cannot be directly quantified; however, future randomized studies comparing antiplatelet and anticoagulation therapy in ESUS patients based on abnormal MOCHA and/or left atrial cardiopathy will clarify optimal management for these patients. Lastly, the study was limited by a small sample size of the historical cohort.

\section{CONCLUSION}

Compared to antiplatelet therapy, anticoagulation therapy may reduce recurrent strokes in the subset of patients with ESUS who have evidence of abnormal coagulation and hemostatic

\section{REFERENCES}

1. Diener H-C, Sacco RL, Easton JD, Granger CB, Bernstein RA, Uchiyama S, et al. Dabigatran for prevention of stroke after embolic stroke of undetermined source. N Engl J Med. (2019) 380:1906-17. doi: 10.1056/NEJMoa181 3959

2. Healey JS, Gladstone DJ, Swaminathan B, Eckstein J, Mundl H, Epstein $\mathrm{AE}$, et al. Recurrent stroke with rivaroxaban compared with aspirin according to predictors of atrial fibrillation: secondary analysis of the NAVIGATE ESUS randomized clinical trial. JAMA Neurol. (2019) 76:764-73. doi: 10.1001/jamaneurol.2019.0617

3. Hart RG, Sharma M, Mundl H, Kasner SE, Bangdiwala SI, Berkowitz SD, et al. Rivaroxaban for stroke prevention after embolic stroke of undetermined source. N Engl J Med. (2018) 378:2191-201. doi: 10.1016/j.jvsv.2018.10.009 activity or LAE. A future study utilizing MOCHA profile and left atrial indices could also modify the diagnostic recommendations for ESUS, which can help identify occult malignancies and undiagnosed atrial fibrillation.

\section{DATA AVAILABILITY STATEMENT}

The original contributions presented in the study are included in the article/Supplementary Material, further inquiries can be directed to the corresponding author/s.

\section{ETHICS STATEMENT}

The studies involving human participants were reviewed and approved by Institutional Review Board of Emory University. Written informed consent for participation was not required for this study in accordance with the national legislation and the institutional requirements.

\section{AUTHOR CONTRIBUTIONS}

KP: wrote first draft of the manuscript and contributed to manuscript revision, read, and approved the submitted version. EM: performed the statistical analysis, wrote sections of the manuscript and contributed to manuscript revision, read, and approved the submitted version. ML: organized the database, performed the statistical analysis and contributed to manuscript revision, read, and approved the submitted version. DE: organized the database, contributed to manuscript revision, read, and approved the submitted version. SR, AD, SB, TB, and LH: contributed to manuscript revision, read, and approved the submitted version. FN: organized the database, contributed to conception and design of the study, wrote sections of the manuscript, and contributed to manuscript revision, read, and approved the submitted version. All authors contributed to the article and approved the submitted version.

\section{SUPPLEMENTARY MATERIAL}

The Supplementary Material for this article can be found online at: https://www.frontiersin.org/articles/10.3389/fneur. 2021.695378/full\#supplementary-material 
stroke. Medicine. (2018) 97:e13830. doi: 10.1097/MD.00000000000 13830

8. Diener H-C, Chutinet A, Easton JD, Granger CB, Kleine E, Marquardt L, et al. Dabigatran or aspirin after embolic stroke of undetermined source in patients with patent foramen ovale: results from RE-SPECT ESUS. Stroke. (2021) 52:1065-8. doi: 10.1161/STROKEAHA.120.031237

9. Lattanzi S, Rinaldi C, Pulcini A, Corradetti T, Angelocola S, Zedde ML, et al. Clinical phenotypes of embolic strokes of undetermined source. Neurol Sci. (2021) 42:297-300. doi: 10.1007/s10072-020-04700-2

10. Sato N, Matsuo R, Kiyuna F, Nakamura K, Hata J, Wakisaka Y, et al. Anticoagulation and risk of stroke recurrence in patients with embolic stroke of undetermined source having no potential source of embolism. Cerebrovasc Dis. (2020) 49:601-8. doi: 10.1159/000510773

11. Jordan K, Yaghi S, Poppas A, Chang AD, Mac Grory B, Cutting S, et al. Left atrial volume index is associated with cardioembolic stroke and atrial fibrillation detection after embolic stroke of undetermined source. Stroke. (2019) 50:1997-2001. doi: 10.1161/STROKEAHA.119.025384

12. Kamel H, Longstreth WT, Tirschwell DL, Kronmal RA, Broderick JP, Palesch YY, et al. The AtRial cardiopathy and antithrombotic drugs in prevention after cryptogenic stroke randomized trial: Rationale and methods. Int J Stroke. (2019) 14:207-14. doi: 10.1177/1747493018799981
13. Shoamanesh A, Hart RG, Connolly SJ, Kasner SE, Smith EE, Martí-Fàbregas J, et al. Microbleeds and the effect of anticoagulation in patients with embolic stroke of undetermined source: an exploratory analysis of the NAVIGATE ESUS randomized clinical trial. JAMA Neurol. (2021) 78:11-20. doi: 10.1001/jamaneurol.2020.3836

Conflict of Interest: FN has a patent on the use of MOCHA to guide medical treatment in cardiovascular disease and stroke.

The remaining authors declare that the research was conducted in the absence of any commercial or financial relationships that could be construed as a potential conflict of interest.

Copyright (C) 2021 Patel, Mikhael, Liu, Rangaraju, Ellis, Duncan, Belagaje, Belair, Henriquez and Nahab. This is an open-access article distributed under the terms of the Creative Commons Attribution License (CC BY). The use, distribution or reproduction in other forums is permitted, provided the original author(s) and the copyright owner(s) are credited and that the original publication in this journal is cited, in accordance with accepted academic practice. No use, distribution or reproduction is permitted which does not comply with these terms. 